"This document is the Accepted Manuscript version of a Published Work that appeared in final form in JOC copyright American Chemical Society after peer review and technical editing by the publisher. To access the final edited and published work see [insert ACS Articles on Request authordirected link to Published Work, see http://pubs.acs.org/doi/abs/10.1021/acs.joc.6b01070"

\title{
Substitution Effects of Hypervalent Iodine(III) Reagents in the
}

\section{Diamination of Styrene}

R. Martín Romero, ${ }^{\dagger}$ José A. Souto ${ }^{\dagger}$ and Kilian Muñiz ${ }^{\dagger+*}$

†Institute of Chemical Research of Catalonia (ICIQ), The Barcelona Institute of Science and Technology, Av. Països Catalans 16, E-43007 Tarragona (Spain)

${ }^{\ddagger}$ Catalan Institution for Research and Advanced Studies (ICREA)

Pg. Lluís Companys 23, 08010 Barcelona (Spain)

*Corresponding Autor. Email: kmuniz@iciq.es

\section{Abstract Graphic/ToC}

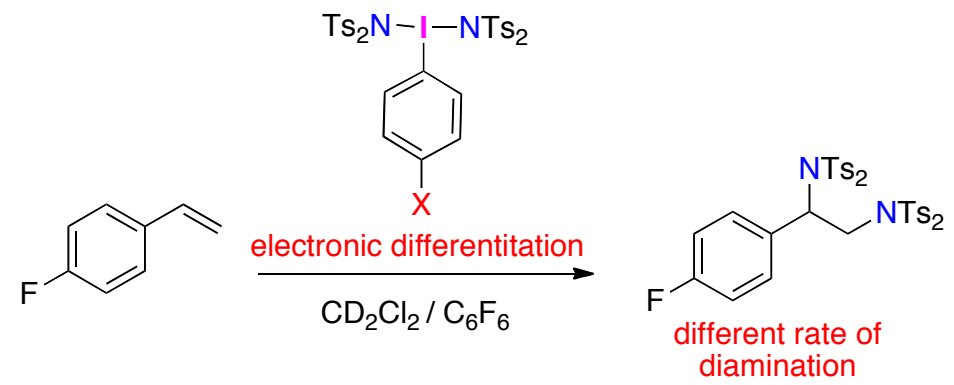

\begin{abstract}
The influence of electronic parameters on the reaction performance of hypervalent iodine(III) reagents in the vicinal diamination of styrene has been investigated. It demonstrates an influence of the relative electron density on the aryl-substituent of the hypervalent iodine reagent. In these cases, compounds with donor substituents outperform
\end{abstract}


the corresponding acceptor-substituted systems. In line with this observation, a rapid enantioselective diamination was observed for a preformed chiral bisimidoiodine(III) reagent. For the first time, X-ray structural data could be obtained for an isolated chiral bisimidoiodine(III).

Hypervalent iodine(III) reagents represent versatile tools in modern organic oxidation chemistry, ${ }^{1}$ and in the development of new synthetic methodology have recently started to challenge traditional procedures based on transition metals. ${ }^{2,3}$

Recently, we introduced monomeric hypervalent iodine(III) compounds bearing defined iodine-nitrogen bonds with bissulfonylimides. As exemplified for bistosylimide, these compounds are best prepared through protonolysis events starting from (diacetoxy)iodobenzene $\mathrm{PhI}(\mathrm{OAc})_{2}$ 1a (Scheme 1) ${ }^{4,5}$ In these cases, treatment of 1a with an equimolar amount of $\mathrm{HNTs}_{2}$ formed the mixed reagent 2a in an irreversible manner. The same reaction in the presence of water forms the $\mu$-oxo-bridged dimer $\mathbf{3 a}$, which can also be accessed from 2a upon controlled partial hydrolysis. Finally, saturation of the coordination sphere of iodine by introduction of an additional $\mathrm{NTs}_{2}$ moiety generates the bisimido species $\mathbf{4 a}$ a $^{4,6,7}$

Successful applications of these aminating reagents involve allylic, ${ }^{8,9}$ acetylenic, ${ }^{10}$ and aromatic $^{11,12}$ amination events and the diamination of alkenes. ${ }^{13}$ The overall series culminated in an enantioselective diamination of styrenes, ${ }^{14}$ which is of unsurpassed efficiency in the field. ${ }^{15,16}$ 


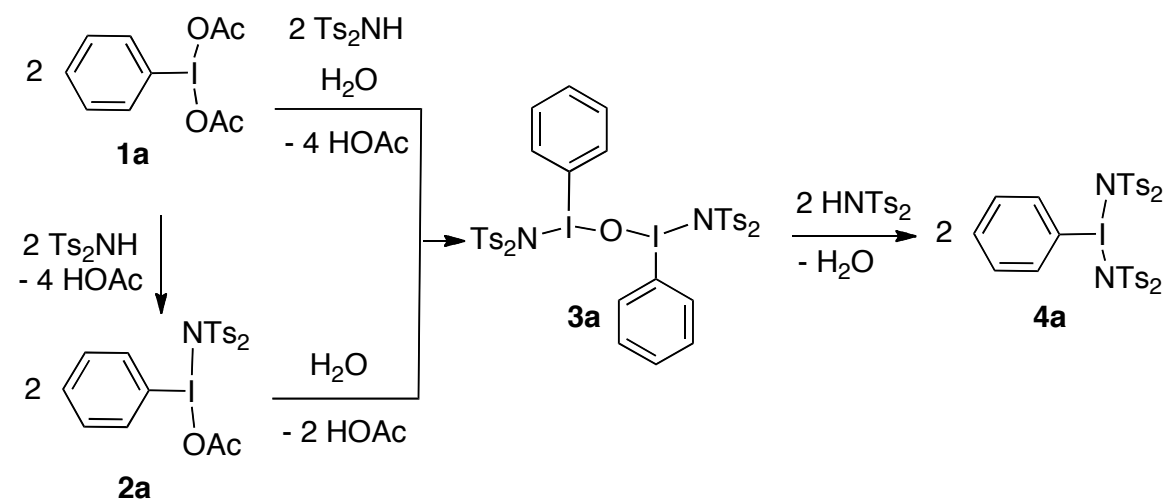

Scheme 1. The phenyliodine(III)/bistosylimide pathways to aminating reagents.

For the simplest case of bismesylimide, a recent theoretical study has uncovered some details regarding the amination pathways involved in styrene amination using hypervalent iodine reagents. ${ }^{17}$ We here report the effect of substituents on the arene ring of the central iodine(III), which gives additional insight into the mechanistic scenario and confirms the general trends from the theoretical study.

Regarding the diamination of styrene 5a with an equimolar amount of the bistosylimide reagent $\mathbf{4 a}$, control experiments revealed a first order dependence on iodine(III) reagent $\mathbf{4 a}$, but a zero-order dependence on the alkene substrate (Scheme 2). ${ }^{18}$ In order to gain a better understanding of this result, four derivatives $\mathbf{4 b - e}$ with the 4-substituted aryliodine(III) core were prepared (Scheme 3). Following our literature protocol, ${ }^{4}$ their synthesis comprises the initial formation of the corresponding dimeric $\mu$-oxo-bridged iodine(III) derivatives $\mathbf{3 b}$-e, which were obtained quantitatively. Upon protonolysis with bistosylimide, compounds $\mathbf{3 b -}$ e cleanly convert into the corresponding monomeric reagents $4 \mathbf{b}-\mathbf{e}$ in quantitative yields. Alternatively, these reagents $4 \mathbf{b}$-e can be equally accessed in a single transformation directly from $\mathbf{1 b - e .}$ 


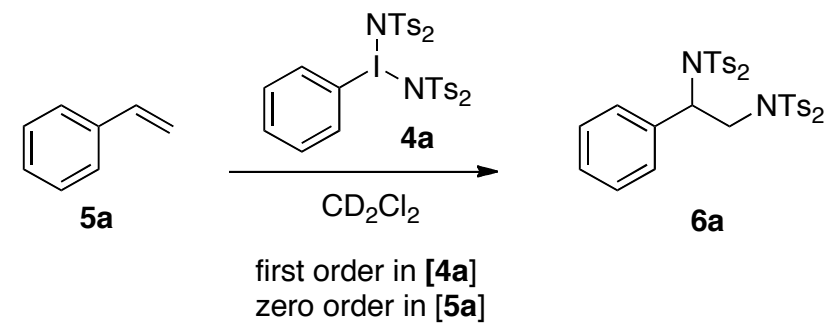

Scheme 2. Dependence of the diamination on styrene 5a and on iodine reagent $4 \mathbf{a}$.

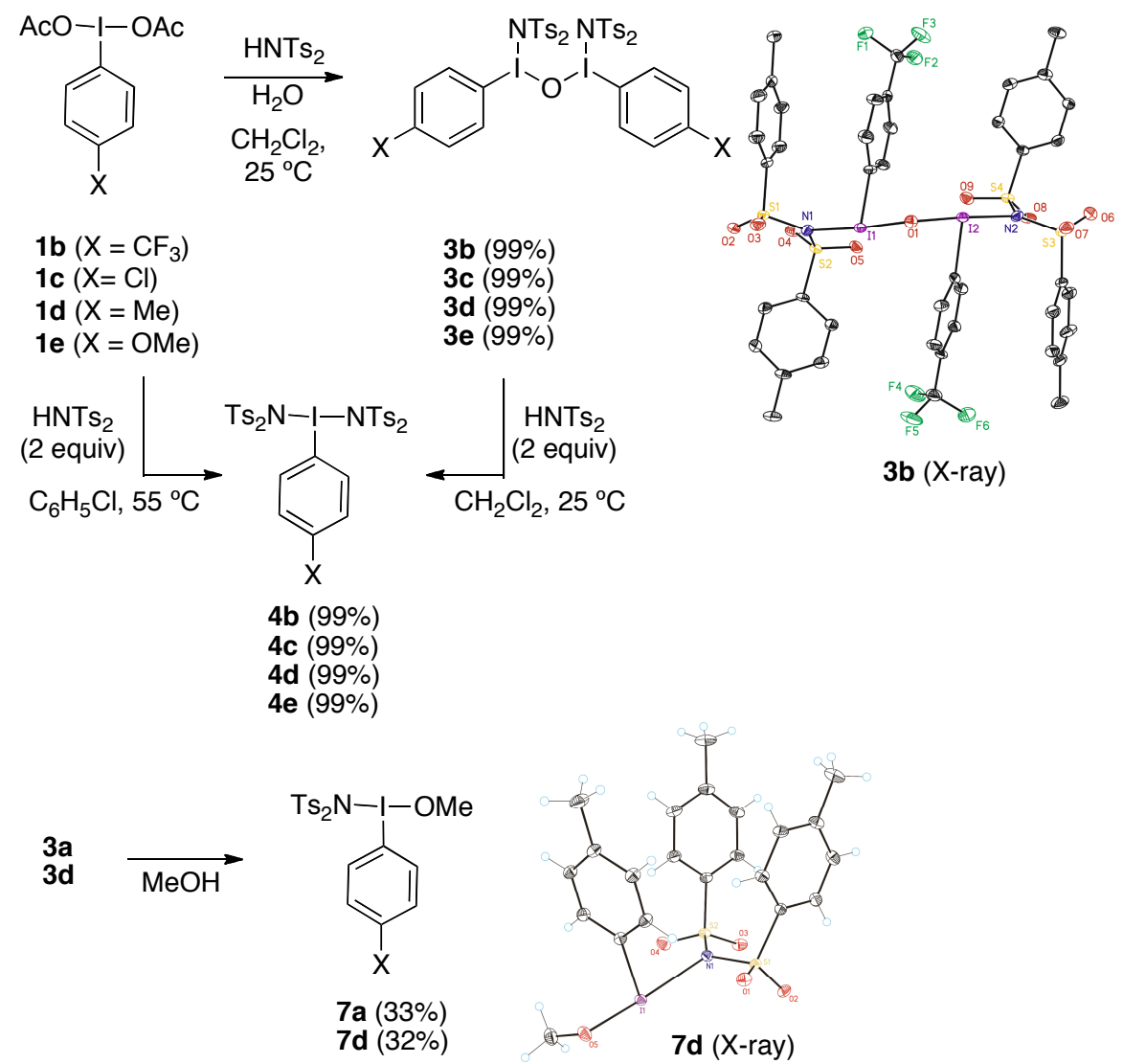

Scheme 3. Synthesis of 4-substituted aryliodine(III) derivatives. 
The expected structures of compounds $\mathbf{3 b}$-e and $\mathbf{4 b}$-e match with the ones for the parent series from Scheme 1. This was further assured from an X-ray analysis of $\mathbf{3 b}{ }^{18}$ Electronic effects render compound $\mathbf{4 d}$ and particularly $\mathbf{4 e}$ less stable than the parent compound $\mathbf{4 a}$ as they are sensitive to hydrolysis. While the corresponding degradation products could not be characterized, the related reaction upon dissolution of compound $\mathbf{4 d}$ in methanol was determined to provide the methoxy derivative $\mathbf{7 d}$. Starting from compounds 3a,d, the expected new mixed iodine(III) derivatives $\mathbf{7 a}, \mathbf{d}$, incorporating a single bistosylimido and a methoxy substituent, were obtained and their structure was confirmed for $\mathbf{7 d}$ by X-ray analysis. ${ }^{20}$ These compounds are remarkably stable and serve as precursor to reactive imidoiodine(III) species upon treatment with bistosylimide.

With these compounds in hand, the reactivity of compounds 4a-e in the diamination of 4fluorostyrene $\mathbf{5 b}$ in dichloromethane was monitored by ${ }^{19} \mathrm{~F}$ NMR with hexafluorobenzene as internal standard (Figure 1).
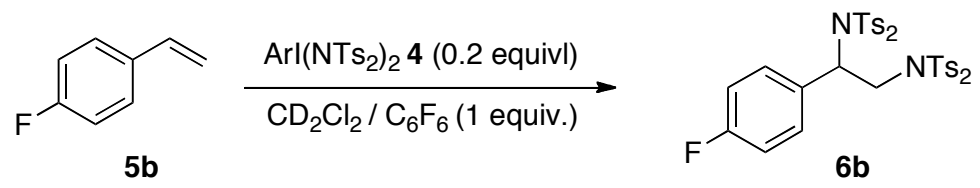


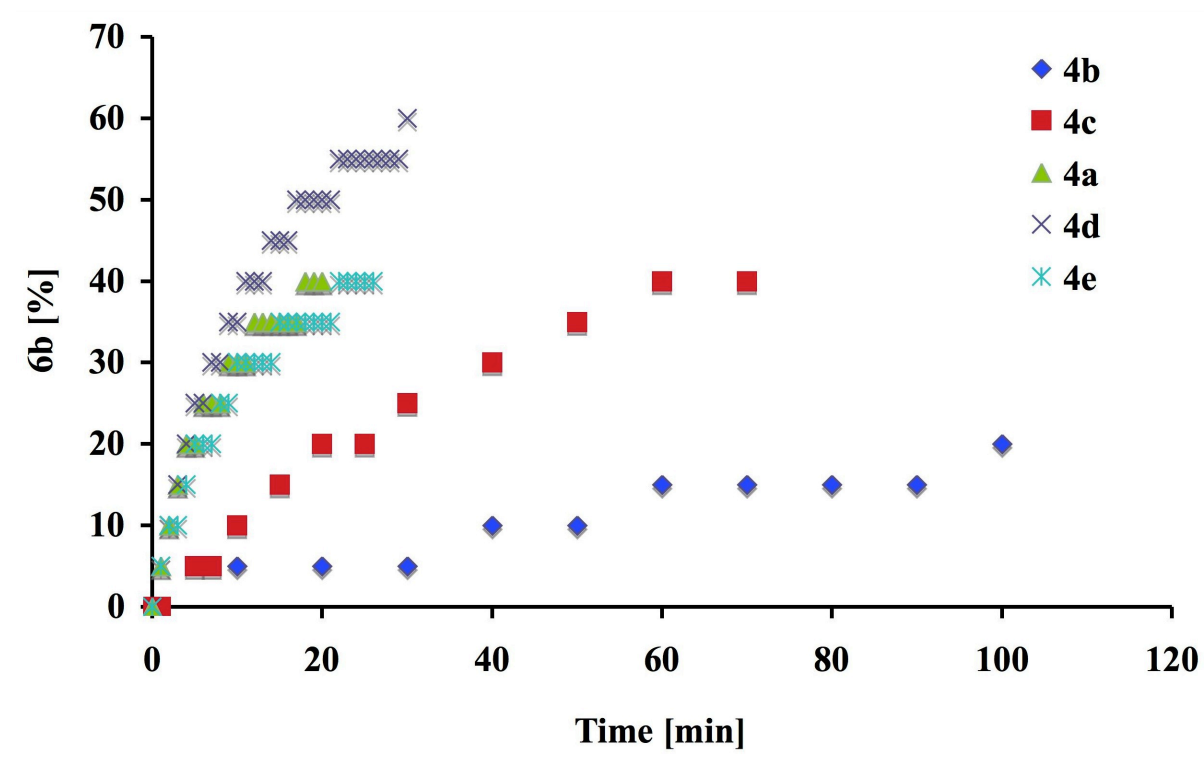

Figure 1. Kinetic profiles for diamination of $\mathbf{5 b}$ with iodine(III) reagents $4 \mathbf{a}$-e.

While the highest rate was observed for the 4-methyl derivative $\mathbf{4 d}$, the corresponding initial rates for $\mathbf{4 a}$ and $\mathbf{4 e}$ were almost identical. In fact, the corresponding Hammett plot shows almost identical outcome for donor-substitution. ${ }^{18}$ This matches with a recent screening on catalyst structure for enantioselective diacetoxylation of alkenes, in which a 4methyl substituent enhanced the rate of the catalysis. ${ }^{19}$ In contrast, electron-deficient derivatives 4b,c showed significantly lower conversion within a comparable reaction period.

These results are instructive as they present an unexpected electronic dependence. One expects a kinetic preference for electron-withdrawing substituents, which enhance the electrophilicity of the iodine(III) center in the initial alkene coordination and in the step of the reductive elimination, where the nucleophuge character of the iodine(III) ${ }^{20}$ dominates the scenario. Instead, it appears that the reaction is accelerated by donor-substitution. Based on the observations from Scheme 2 and Figure 1, we now propose that the decisive step in 
the diamination of alkenes with bistosylimide as nitrogen source consists of a dissociative pre-equilibrium (Figure 2). Previously, this dissociation context had been investigated experimentally for the neutral species $\mathbf{4 a},{ }^{4}$ and had been identified by theory as the energetically most demanding step of the diamination reactions of styrene with related bismesylimide. ${ }^{17}$ The data presented here imply that the enhanced acceleration with $\mathbf{4 d}$ is based on an electronic donor stabilization of the involved cationic intermediate $4+$. The relation between donor substituent and overall rate needs to be balanced in a way that the electrophilic character at iodine is not reduced too much due to electronic stabilization as appears to be the case for the 4-methoxy substituent.

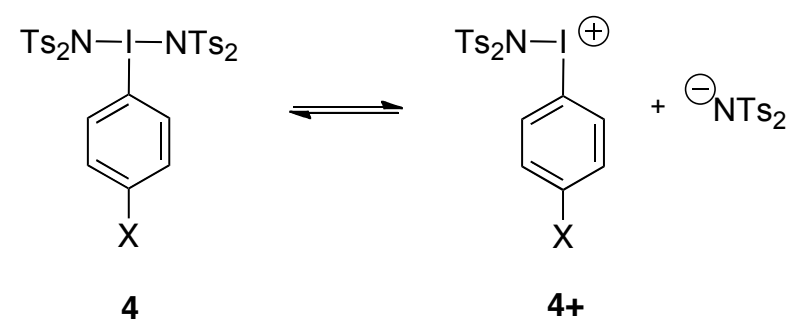

Figure 2. Electronic influence on I-N bond dissociation in reagents 4.

We then turned our attention toward the structures of chiral aryl iodides. Regarding the scenario of chirality, we had previously employed a chiral $\mathrm{C}_{2}$-symmetric hypervalent iodine reagent bearing two lactate side chains in the enantioselective diamination of styrenes. ${ }^{14 a}$ In these cases, bismesylimide served as the best nitrogen source by providing the highest enantiomeric excesses of the final diamine products. Despite repeated attempts, we were unable to obtain any evidence for the incorporation of the bissulfonylimides into the coordination sphere of the iodine(III) center. Attempts were further hampered by the low solubility of the involved species. However, the anticipated sequential exchange of acetate 
for bistosylimide could be accomplished for the case of the chiral iodine(III) compound $\mathbf{1 f}^{21 \mathrm{a}, \mathrm{b}}$ bearing a single lactic ester side chain (Scheme 4). As in the case of the achiral derivatives 1a-e, sequential replacement took place to initially generate the $\mu$-oxo-bridged compound 3f. The structure of $\mathbf{3 f}$ was confirmed unambiguously by single crystal X-ray analysis. ${ }^{18}$ Its conversion into the bisimido derivative $\mathbf{4 f}$ under standard protonolysis with free bistosylimide was possible, but did not proceed in quantitative manner. Instead, compound $\mathbf{4 f}$ was better accessed through direct acetate for imide exchange at stage 1f. It represents only the second iodine(III)-nitrogen derivative of a chiral iodine(III) reagent ${ }^{14 \mathrm{~b}}$ and the first for the successful class of lactate derivatives. ${ }^{21}$

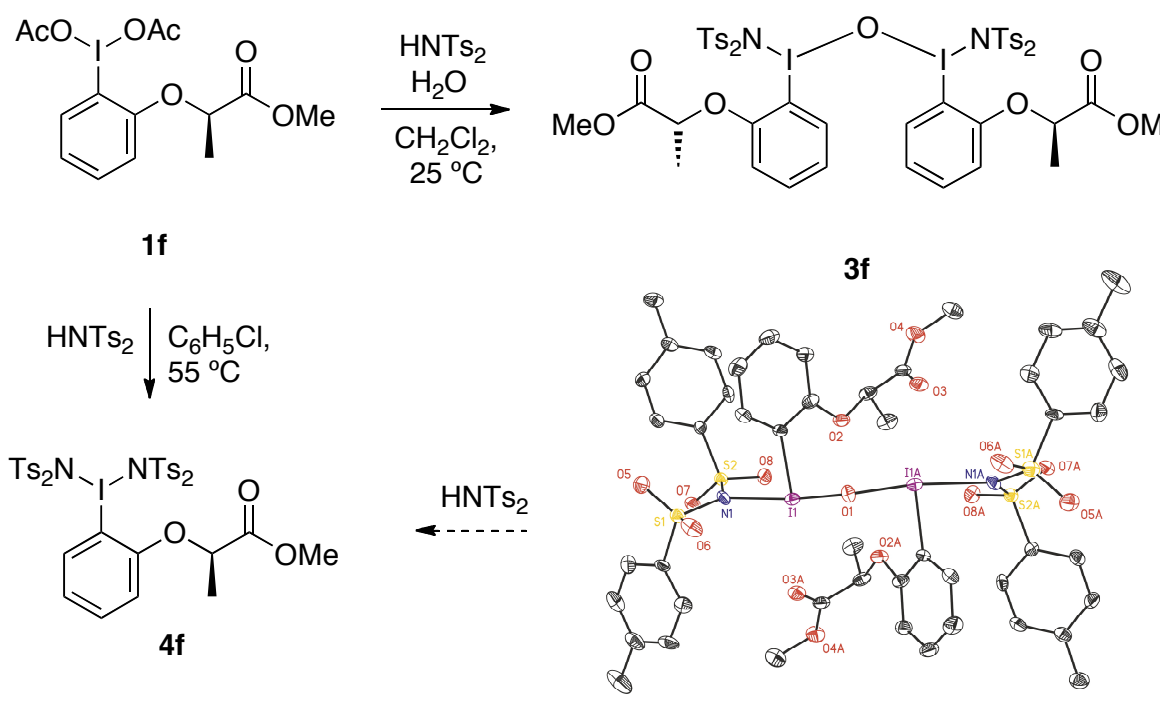

Scheme 4. Synthesis of enantiopure iodine(III) reagents with I-N bond.

Kinetic experiments reveal that $\mathbf{4 f}$ reacts with 4 -fluoro styrene in a rapid conversion leading to $50 \%$ diamination product $\mathbf{6 b}$ within only 8 minutes reaction time. ${ }^{18}$ Although this product is formed with a low $24 \%$ ee, this rate effect, which is attributed to the electronically favorable 2-oxygenated substituent, is remarkable. It compares well with the observed rapid rate for related bislactamide derivatives, which were employed as catalysts 
in the homogeneous diacetoxylation of styrenes and which could effectively override potential background reactions from the terminal peracid oxidant. ${ }^{19}$ For styrene itself, $\mathbf{4 f}$ represents the active and only required reagent in the diamination reaction ${ }^{16 a}$ as demonstrated by the chemoselective formation of $6 \mathbf{a}$ (Scheme 5). Here, the $(S)$-configured product is formed with $33 \%$ ee at a reaction temperature of $0{ }^{\circ} \mathrm{C}$, while the corresponding in situ-formed reagent from bislactate 8 generates $(\boldsymbol{S})-\mathbf{6 a}$ with $80 \%$ ee. This value is comparable to reaction results at a slightly higher temperature as documented previously. ${ }^{16 a}$ In comparing the enantioinductions obtained with $\mathbf{4 f}$ and $\mathbf{8}$, the outcome suggests that ultimately both lactates within reagent $\mathbf{8}$ are required for high enantiocontrol in the diamination and confirms that the bislactate motif represents the currently most promising chiral iodine(III) reagent class. ${ }^{14 a, 19,21,22}$
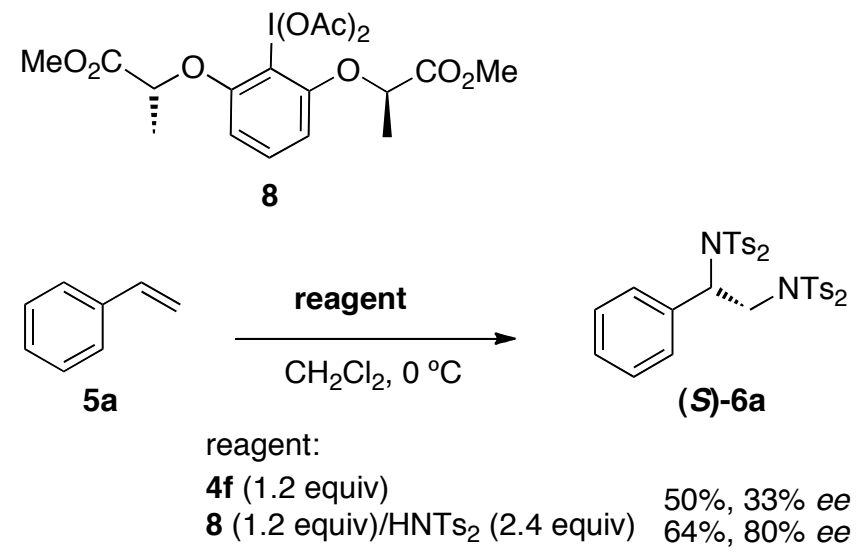

Scheme 5. Enantioselective diamination of styrene.

In summary, we have succeeded in the synthesis and isolation of various structurally and electronically diversified hypervalent iodine(III) reagents. These compounds have led to two key insights, which round up the development of intermolecular amination reactions 
with hypervalent iodine(III) reagents. First, the diamination of styrenes with bistosylimides as nitrogen sources proceeds through a rate-determining preequilibrium with the formation of a cationic iodine(III) reagent that engages in styrene coordination as predicted from a theoretical investigation. ${ }^{17}$ Related studies on a chiral, non-racemic iodine(III) derivative for the first time have allowed the isolation of reagents with defined iodine-nitrogen bonds. These compounds lend weight to the assumption that enantioselective amination processes such as the diamination of styrenes under the previously established in situ conditions involve the initial formation of imidoiodine(III) species.

\section{Experimental Section}

\section{General Remarks}

Chemicals and solvents for chromatography were used as received. Solvents were obtained from a solvent purification system. Reactions that were monitored by TLC were visualized by a dual short wave/long wave UV lamp. ${ }^{1} \mathrm{H},{ }^{13} \mathrm{C}$ and ${ }^{19} \mathrm{~F}$ NMR spectra were recorded using an internal deuterium lock on a 300 or a $500 \mathrm{MHz}$ spectrometer. All chemical shifts in NMR experiments are reported as ppm downfield from TMS. $J$ couplings are reported in Hz. The following calibrations were used: $\mathrm{CDCl}_{3} \delta=7.26$ and 77.0 ppm, DMSO- $d_{6} \delta=$ 2.50 and $39.5 \mathrm{ppm}$. Infrared spectra were recorded on a FT-IR fitted with an ATR accessory. Absorptions are given in wavenumbers $\left(\mathrm{cm}^{-1}\right)$. High resolution mass spectra were obtained on a HRMS-TOF spectrometer.

General procedure for synthesis of $\mu$-oxo-bis[(4-methyl)- $N$ tosylbenzenesulfonamidyl(aryl)iodine(III)] 3. To a solution of the corresponding (diacetoxyiodo)arene 1 (0.3 mmol, 2 equiv.) in $\mathrm{CH}_{2} \mathrm{Cl}_{2}(0.6 \mathrm{~mL}), \mathrm{HNTs}_{2}(0.3 \mathrm{mmol}, 2$ equiv.) was added in one portion and the reaction mixture was stirred at room temperature 
overnight. Then, cyclohexane was added and the solvent was removed under reduced pressure. Twice more, $\mathrm{CH}_{2} \mathrm{Cl}_{2}$ and cyclohexane were added and removed under reduced pressure. After drying at reduced pressure, the corresponding iodine(III) compound was obtained in quantitative yield.

General procedure for synthesis of $\operatorname{Bis[(4-methyl)-N\text {-}}$ tosylbenzenesulfonamidyl]iodosoarene 4. To a solution of the corresponding (diacetoxyiodo)arene 1 ( $0.3 \mathrm{mmol}, 1$ equiv.) in chlorobenzene $(1.5 \mathrm{~mL}), \mathrm{HNTs}_{2}(0.6 \mathrm{mmol}$, 2 equiv.) was added and the reaction mixture was stirred at $55^{\circ} \mathrm{C}$. The solvent was removed under reduced pressure. Three times, chlorobenzene was added and removed under reduced pressure. After drying at reduced pressure, the corresponding iodine(III) compound was obtained in quantitative yield.

General procedure for synthesis of methoxy((4-methyl)- $N$ tosylbenzenesulfonamidyl)iodosoarene 7. To a solution of diacetoxyiodoarene 1 (1 mmol, 1 equiv.) in chlorobenzene (3.7 mL) and $\mathrm{H}_{2} \mathrm{O}$ (1 equiv.), $\mathrm{HNTs}_{2}$ (1 equiv.) was added and the reaction mixture was stirred at $55{ }^{\circ} \mathrm{C}$. The solvent was removed under reduced pressure. Three times, chlorobenzene was added removed under reduced pressure. After drying at reduced pressure, the corresponding iodine(III) compound $\mathbf{3}$ was obtained as a yellow solid. To this solid, $\mathrm{MeOH}$ was added until a white suspension formed at $4{ }^{\circ} \mathrm{C}$. Filtration provided the title compounds in pure form.

Kinetic measurement of diamination reaction with diferent iodine(III) reagents 4. In a NMR tube, the respective reagent $\operatorname{ArI}\left(\mathrm{NTs}_{2}\right)_{2} 4$ (1 equiv.), $\mathrm{C}_{6} \mathrm{~F} 6$ (5 equiv.) and 4-fluorostyrene $5 \mathbf{b}$ 
(0.2 mmol, 5 equiv.) were dissolved in $\mathrm{CD}_{2} \mathrm{Cl}_{2}(0.6 \mathrm{~mL})$. The reaction progress was monitored by ${ }^{19} \mathrm{~F}$ NMR spectroscopy recording individual spectra within a time interval of 1 minute.

$\mu$-Oxo-bis[(4-methyl)- $N$-tosylbenzenesulfonamidyl( $p$-trifluoromethyl-phenyl)iodine(III)] (3b). White solid (0.181 g, $0.15 \mathrm{mmol}, 99 \%) .{ }^{1} \mathrm{H}$ NMR (400 MHz, DMSO-d $): \delta=2.32(\mathrm{~s}$, $12 \mathrm{H}), 7.15(\mathrm{~d}, J=8.0 \mathrm{~Hz}, 8 \mathrm{H}), 7.52(\mathrm{~d}, J=8.1 \mathrm{~Hz}, 8 \mathrm{H}), 7.99(\mathrm{~d}, J=8.3 \mathrm{~Hz}, 4 \mathrm{H}), 8.39(\mathrm{~d}, J$ $=8.1 \mathrm{~Hz}, 4 \mathrm{H}) \cdot{ }^{13} \mathrm{C}$ NMR $\left(100 \mathrm{MHz}, \mathrm{DMSO}-d_{6}\right): \delta=20.8,123.4(\mathrm{q}, J=272 \mathrm{~Hz}), 126.2$, $127.9(\mathrm{q}, J=4 \mathrm{~Hz}), 128.3,131.9(\mathrm{q}, J=33 \mathrm{~Hz}), 134.7,138.2,139.8,143.3 .{ }^{19} \mathrm{~F}-\mathrm{NMR}(376$ MHz, DMSO-d $): \delta=-61.3$. IR $v\left(\mathrm{~cm}^{-1}\right): 3095,3048,2924,1595,1398,1335,1316,1142$ 1081, 1065, 938. HRMS (MALDI+): calc. for $\left[\mathrm{C}_{21} \mathrm{H}_{18} \mathrm{~F}_{3} \mathrm{INO}_{4} \mathrm{~S}_{2}\right]^{+}$: 595.9668; found: 595.9671. m.p.: $147-148^{\circ} \mathrm{C}$.

$\operatorname{Bis}[(4-m e t h y l)-N$-tosylbenzenesulfonamidyl]iodoso- $p$-trifluoromethylbenzene (4b). White solid (0.276 mg, $0.3 \mathrm{mmol}, 99 \%) .{ }^{1} \mathrm{H}$ NMR (400 MHz, DMSO- $\left.d_{6}\right): \delta=2.33(\mathrm{~s}, 12 \mathrm{H}), 7.18$ $(\mathrm{d}, J=8.0 \mathrm{~Hz}, 8 \mathrm{H}), 7.54(\mathrm{~d}, J=8.1 \mathrm{~Hz}, 8 \mathrm{H}), 7.99(\mathrm{~d}, J=8.4 \mathrm{~Hz}, 2 \mathrm{H}), 8.39(\mathrm{~d}, J=8.3 \mathrm{~Hz}$ 2H). ${ }^{13} \mathrm{C}$ NMR (125 MHz, DMSO- $\left.d_{6}\right): \delta=20.9,123.5$ (q, $\left.J=273 \mathrm{~Hz}\right), 126.5,127.8,128.7$, $131.9(\mathrm{q}, J=33 \mathrm{~Hz}), 134.9,138.3,141.3,141.4 .{ }^{19} \mathrm{~F}-\mathrm{NMR}\left(376 \mathrm{MHz}, \mathrm{DMSO}-d_{6}\right): \delta=-$ 61.3. IR $v\left(\mathrm{~cm}^{-1}\right): 3157,3097,1595,1493,1448,1399,1359,1336,1316,1164,1143,1082$, 1065, 1044, 1020, 999. HRMS (MALDI+): calc. for $\left[\mathrm{C}_{21} \mathrm{H}_{18} \mathrm{~F}_{3} \mathrm{INO}_{4} \mathrm{~S}_{2}\right]^{+}:$595.9668; found: 595.9705. m.p.: $162-165^{\circ} \mathrm{C}$. 
$\mu$-Oxo-bis[(4-methyl)- $N$-tosylbenzenesulfonamidyl(p-chloro-phenyl)iodine(III)]

$(3 c)$.

White solid (0.171 g, $0.15 \mathrm{mmol}, 99 \%) .{ }^{1} \mathrm{H}$ NMR (400 MHz, DMSO- $\left.d_{6}\right): \delta=2.31$ (s, 12H), $7.14(\mathrm{~d}, J=7.8 \mathrm{~Hz}, 8 \mathrm{H}), 7.52(\mathrm{~d}, J=7.8 \mathrm{~Hz}, 8 \mathrm{H}), 7.68(\mathrm{~d}, J=8.0 \mathrm{~Hz}, 4 \mathrm{H}), 8.20(\mathrm{~d}, J=7.6$ $\mathrm{Hz}, 4 \mathrm{H}) .{ }^{13} \mathrm{C}$ NMR $\left(100 \mathrm{MHz}, \mathrm{DMSO}-d_{6}\right): \delta=20.9,121.4,126.2,128.2,131.2,136.3$, 137.4, 139.6, 143.8. IR $v\left(\mathrm{~cm}^{-1}\right): 3089,2919,1596,1494,1469,1390,1332,1306,1288$, 1143, 1082, 1020, 997. HRMS (MALDI+): calc. for $\left[\mathrm{C}_{20} \mathrm{H}_{18} \mathrm{ClNO}_{4} \mathrm{~S}_{2} \mathrm{I}\right]^{+}: 561.9405$; found: 561.9368. m.p.: $169-170^{\circ} \mathrm{C}$.

$\operatorname{Bis}[(4-m e t h y l)-N$-tosylbenzenesulfonamidyl]iodoso- $p$-chlorobenzene (4c). White solid (0.266 mg, $0.3 \mathrm{mmol}, 99 \%) .{ }^{1} \mathrm{H}$ NMR (400 MHz, DMSO- $\left.d_{6}\right): \delta=2.33(\mathrm{~s}, 12 \mathrm{H}), 7.18(\mathrm{~d}, J$ $=7.9 \mathrm{~Hz}, 8 \mathrm{H}), 7.54(\mathrm{~d}, J=8.1 \mathrm{~Hz}, 8 \mathrm{H}), 7.69(\mathrm{~d}, J=8.6 \mathrm{~Hz}, 2 \mathrm{H}), 8.22(\mathrm{~d}, J=8.7 \mathrm{~Hz}, 2 \mathrm{H})$. ${ }^{13} \mathrm{C}$ NMR $\left(100 \mathrm{MHz}, \mathrm{DMSO}-d_{6}\right): \delta=20.9,126.3,128.5,130.6$ 131.2, 136.3, 138.9, 140.6, 142.2. IR $v\left(\mathrm{~cm}^{-1}\right): 3156,2921,1596,1494,1469,1359,1334,1305,1164,1143,1082$, 1019, 998. HRMS (MALDI+): calc. for $\left[\mathrm{C}_{20} \mathrm{H}_{18} \mathrm{ClINO}_{4} \mathrm{~S}_{2}\right]^{+}: 561.9405$; found: 561.9379 . m.p.: $154-156^{\circ} \mathrm{C}$.

$\mu$-Oxo-bis[(4-methyl)- $N$-tosylbenzenesulfonamidyl( $p$-methyl-phenyl)iodine(III)] $(3 d)$. White solid (0.165 g, $0.15 \mathrm{mmol}, 99 \%) .{ }^{1} \mathrm{H}$ NMR (400 MHz, DMSO- $\left.d_{6}\right): \delta=2.31$ (s, 12H), $2.42(\mathrm{~s}, 6 \mathrm{H}), 7.14(\mathrm{~d}, J=7.9 \mathrm{~Hz}, 8 \mathrm{H}), 7.41(\mathrm{~d}, J=8.7 \mathrm{~Hz}, 4 \mathrm{H}), 7.52(\mathrm{~d}, J=8.0 \mathrm{~Hz}, 8 \mathrm{H})$, $8.10(\mathrm{~d}, J=8.7 \mathrm{~Hz}, 4 \mathrm{H}) .{ }^{13} \mathrm{C}$ NMR $\left(100 \mathrm{MHz}, \mathrm{DMSO}-d_{6}\right): \delta=20.9,21.1,120.2,126.1$, 128.2, 131.7, 134.8, 139.5, 143.1, 143.8. IR $v\left(\mathrm{~cm}^{-1}\right): 3029,2919,1596,1493,1399,1329$, 1305, 1288, 1207, 1187, 1159, 1143, 1119, 1082, 1020, 1000, 956. HRMS (MALDI+): calc. for $\left[\mathrm{C}_{21} \mathrm{H}_{21} \mathrm{INO}_{4} \mathrm{~S}_{2}\right]^{+}: 541.9951$; found: 541.9922 . m.p.: 160-161 ${ }^{\circ} \mathrm{C}$. 
Bis[(4-methyl)- $N$-tosylbenzenesulfonamidyl]iodoso- $p$-methylbenzene (4d). White solid (0.260 g, $0.3 \mathrm{mmol}, 99 \%) .{ }^{1} \mathrm{H}$ NMR (400 MHz, DMSO- $\left.d_{6}\right): \delta=2.32(\mathrm{~s}, 12 \mathrm{H}), 2.42(\mathrm{~s}, 3 \mathrm{H})$, $7.17(\mathrm{~d}, J=8.0 \mathrm{~Hz}, 8 \mathrm{H}), 7.42(\mathrm{~d}, J=8.1 \mathrm{~Hz}, 2 \mathrm{H}), 7.53(\mathrm{~d}, J=8.2 \mathrm{~Hz}, 8 \mathrm{H}), 8.11(\mathrm{~d}, J=8.3$ $\mathrm{Hz}, 2 \mathrm{H}) .{ }^{13} \mathrm{C}$ NMR $\left(125 \mathrm{MHz}, \mathrm{DMSO}-d_{6}\right): \delta=20.9,21.1,126.4,128.7,131.5,131.8$, 134.9, 136.9, 141.2, 141.4. IR $v\left(\mathrm{~cm}^{-1}\right): 3050,2925,1908,1710,1595,1491,1472,1452$, 1395, 1343, 1326, 1305, 1231, 1207, 1184, 1148, 1120, 1081, 1043, 1017, 993. HRMS (MALDI+): calc. for $\left[\mathrm{C}_{21} \mathrm{H}_{21} \mathrm{INO}_{4} \mathrm{~S}_{2}\right]^{+}: 541.9951$; found: 541.9956. m.p.: $136-141^{\circ} \mathrm{C}$.

$\mu$-Oxo-bis[(4-methyl)- $N$-tosylbenzenesulfonamidyl( $p$-methoxy-phenyl)iodine(III)] (3e). White solid (0.170 g, $0.15 \mathrm{mmol}, 99 \%) .{ }^{1} \mathrm{H}$ NMR (400 MHz, DMSO- $\left.d_{6}\right): \delta=2.31(\mathrm{~s}, 12 \mathrm{H})$, $3.85(\mathrm{~s}, 6 \mathrm{H}), 7.10-7.16(\mathrm{~m}, 12 \mathrm{H}), 7.52(\mathrm{~d}, J=8.1 \mathrm{~Hz}, 8 \mathrm{H}), 8.16(\mathrm{~d}, J=6.8 \mathrm{~Hz}, 4 \mathrm{H}) .{ }^{13} \mathrm{C}$ NMR $\left(100 \mathrm{MHz}, \mathrm{DMSO}-d_{6}\right): \delta=20.9,55.8,113.5,116.7,126.2,128.2,137.5,139.7$, 143.7, 162.3. IR $v\left(\mathrm{~cm}^{-1}\right): 2920,1572,1487,1457,1403,1328,1301,1250,1175,1141$, 1080, 1019, 946. MS (MALDI+): 558.1. m.p.: 132-134 ${ }^{\circ} \mathrm{C}$.

Bis[(4-methyl)- $N$-tosylbenzenesulfonamidyl]iodoso- $p$-methoxybenzene (4e). White solid (0.265 g, $0.3 \mathrm{mmol}, 99 \%) .{ }^{1} \mathrm{H}$ NMR (400 MHz, DMSO- $\left.d_{6}\right): \delta=2.33(\mathrm{~s}, 12 \mathrm{H}), 3.84(\mathrm{~s}, 3 \mathrm{H})$, $7.13(\mathrm{~d}, J=8.8 \mathrm{~Hz}, 2 \mathrm{H}), 7.19(\mathrm{~d}, J=7.8 \mathrm{~Hz}, 8 \mathrm{H}), 7.55(\mathrm{~d}, J=7.9 \mathrm{~Hz}, 8 \mathrm{H}), 8.18(\mathrm{~d}, J=8.7$ $\mathrm{Hz}, 2 \mathrm{H}) .{ }^{13} \mathrm{C}$ NMR $\left(100 \mathrm{MHz}, \mathrm{DMSO}-d_{6}\right): \delta=20.9,55.8,113.5,116.8,126.4,128.6$, 137.6, 140.8, 142.0, 162.4. IR $v\left(\mathrm{~cm}^{-1}\right): 3096,2922,1595,1569,1484,1442,1403,1346$, 1330, 1302, 1253, 1145, 1120, 1081, 1018, 918. MS (MALDI+): 558.1. m.p.: 131-132 ${ }^{\circ} \mathrm{C}$. 
$\mu$-Oxo-bridged chiral iodine(III) (3f). White solid (0.191 g, $0.15 \mathrm{mmol}, 99 \%) .{ }^{1} \mathrm{H}$ NMR (400 MHz, DMSO- $\left.d_{6}\right): \delta=1.63(\mathrm{~d}, J=6.8 \mathrm{~Hz}, 6 \mathrm{H}), 2.31(\mathrm{~s}, 12 \mathrm{H}), 3.72(\mathrm{~s}, 6 \mathrm{H}), 5.30(\mathrm{q}, J=$ $6.8 \mathrm{~Hz}, 2 \mathrm{H}), 7.14-7.16(\mathrm{~m}, 10 \mathrm{H}), 7.24(\mathrm{~d}, J=8.2 \mathrm{~Hz}, 2 \mathrm{H}), 7.52(\mathrm{~d}, J=8.2 \mathrm{~Hz}, 8 \mathrm{H}), 7.62-$ $7.71(\mathrm{~m}, 2 \mathrm{H}), 8.30(\mathrm{~d}, J=7.6 \mathrm{~Hz}, 2 \mathrm{H}) .{ }^{13} \mathrm{C}$ NMR $\left(100 \mathrm{MHz}, \mathrm{DMSO}-d_{6}\right): \delta=18.0,20.9$, $52.5,73.4,113.8,115.4,123.6,126.1,128.2,135.6,138.0,139.6,143.8,154.4,171.0$. IR $v\left(\mathrm{~cm}^{-1}\right): 2953,1736,1651,1586,1569,1493,1470,1448,1385,1360,1326,1282,1248$ 1217, 1186, 1143, 1099, 1080, 1051, 1017, 981. HRMS (MALDI+): calc. for $\left[\mathrm{C}_{24} \mathrm{H}_{25} \mathrm{INO}_{7} \mathrm{~S}_{2}\right]^{+}:$630.0112; found: 630.0131. m.p.: 139-141 ${ }^{\circ} \mathrm{C} .[\alpha]_{\mathrm{D}}{ }^{27}:-290.1(\mathrm{c}=0.10$, $\mathrm{CH}_{2} \mathrm{Cl}_{2}$ ).

Chiral iodine(III) (4f). White solid (0.286 g, $0.3 \mathrm{mmol}, 99 \%){ }^{1} \mathrm{H}$ NMR (400 MHz, DMSO$\left.d_{6}\right): \delta=1.63(\mathrm{~d}, J=6.7 \mathrm{~Hz}, 3 \mathrm{H}), 2.33(\mathrm{~s}, 12 \mathrm{H}), 3.72(\mathrm{~s}, 3 \mathrm{H}), 5.30(\mathrm{q}, J=6.7 \mathrm{~Hz}, 1 \mathrm{H}), 7.13-$ $7.26(\mathrm{~m}, 10 \mathrm{H}), 7.55$ (d, $J=7.9 \mathrm{~Hz}, 8 \mathrm{H}), 7.67$ (t, $J=7.6 \mathrm{~Hz}, 1 \mathrm{H}), 8.30(\mathrm{~d}, J=9.0 \mathrm{~Hz}, 1 \mathrm{H})$. ${ }^{13} \mathrm{C}$ NMR $\left(100 \mathrm{MHz}\right.$, DMSO- $\left.d_{6}\right): \delta=18.0,20.9,52.5,73.4,113.8,115.4,123.6,126.3$, 128.5, 135.6, 138.0, 140.7, 142.2, 154.4, 171.0. IR $v\left(\mathrm{~cm}^{-1}\right): 3155,1738,1595,1493,1469$, 1377, 1356, 1330, 1282, 1216, 1162, 1144, 1081, 1053, 1017, 980. HRMS (MALDI+): calc. for $\left[\mathrm{C}_{24} \mathrm{H}_{25} \mathrm{INO}_{7} \mathrm{~S}_{2}\right]^{+}: 630.0112$; found: 630.0001 . m.p.: $146-147{ }^{\circ} \mathrm{C} .[\alpha]_{\mathrm{D}}{ }^{27}:-233.0(\mathrm{c}$ $=0.10, \mathrm{CH}_{2} \mathrm{Cl}_{2}$ ).

Methoxy((4-methyl)- $N$-tosylbenzenesulfonamidyl)iodosobenzene (7a) White solid (0.185 g, $0.33 \mathrm{mmol}, 33 \%) .{ }^{1} \mathrm{H}$ NMR (400 MHz, DMSO- $\left.d_{6}\right): \delta=2.31(\mathrm{~s}, 6 \mathrm{H}), 3.17(\mathrm{~s}, 3 \mathrm{H}), 7.14$ $(\mathrm{d}, J=8.0 \mathrm{~Hz}, 4 \mathrm{H}), 7.52(\mathrm{~d}, J=8.1 \mathrm{~Hz}, 4 \mathrm{H}), 7.60-7.64(\mathrm{~m}, 2 \mathrm{H}), 7.69-7.73(\mathrm{~m}, 1 \mathrm{H}), 8.22(\mathrm{~d}$, $7.6 \mathrm{~Hz}, 2 \mathrm{H}) .{ }^{13} \mathrm{C}$ NMR $\left(100 \mathrm{MHz}, \mathrm{DMSO}-d_{6}\right): \delta=20.9,63.2,122.1,126.2,128.3,131.4$, 
133.1, 135.5, 139.9, 143.4. IR $v\left(\mathrm{~cm}^{-1}\right): 2919 ; 2813,1595,1493,1469,1446,1334,1148$ 1080, 1019, 989. HRMS (MALDI+): calc. for $\left[\mathrm{C}_{20} \mathrm{H}_{19} \mathrm{INO}_{4} \mathrm{~S}_{2}\right]^{+}:$527.9795; Found: 527.9859. m.p.: $163-167^{\circ} \mathrm{C}$.

Methoxy((4-methyl)- $N$-tosylbenzenesulfonamidyl)-4-methyl-iodosobenzene (7d) White solid (0.184 mg, $0.32 \mathrm{mmol}, 32 \%) .{ }^{1} \mathrm{H}$ NMR (400 MHz, DMSO- $\left.d_{6}\right): \delta=2.32(\mathrm{~s}, 6 \mathrm{H}), 2.42$ (s, 3H), $3.17(\mathrm{~s}, 3 \mathrm{H}), 7.16(\mathrm{~d}, J=8.0 \mathrm{~Hz}, 4 \mathrm{H}), 7.42(\mathrm{~d}, J=8.1 \mathrm{~Hz}, 2 \mathrm{H}), 7.53(\mathrm{~d}, J=8.1 \mathrm{~Hz}$,

4H), $8.11(\mathrm{~d}, J=8.2 \mathrm{~Hz}, 2 \mathrm{H}) .{ }^{13} \mathrm{C}$ NMR $\left(100 \mathrm{MHz}, \mathrm{DMSO}-d_{6}\right): \delta=20.9,21.1,48.6,126.2$, 128.3, 131.5, 131.7, 134.8, 136.9, 139.9, 143.1. IR $v\left(\mathrm{~cm}^{-1}\right): 3026,2957,2921,1597,1482$, $1447,1399,1317,1283,1138,1079,960,805,771,664,547,506,483$. HRMS (MALDI+): calc. for $\left[\mathrm{C}_{21} \mathrm{H}_{21} \mathrm{INO}_{4} \mathrm{~S}_{2}\right]^{+}:$541.9957; Found: 541.9983. m.p.: $132-134{ }^{\circ} \mathrm{C}$.

\section{Supporting Information}

Full experimental description of control experiments and copies of ${ }^{1} \mathrm{H}$ and ${ }^{13} \mathrm{C}$ NMR spectra of new compounds (pdf), and data on X-ray structure determination (cif). This material is available free of charge via the Internet at http://pubs.acs.org.

\section{Acknowledgements}

The authors thank the ICIQ Foundation, the Spanish Ministerio de Economía y Competitividad and FEDER (CTQ2014-56474R grant to K. M., and Severo Ochoa Excellence Accreditation 2014-2018 to ICIQ, SEV-2013-0319) for financial support and Eduardo Escudero-Adán for the X-ray analyses. R. M. R. thanks MEC for an FPU fellowship. 


\section{References}

(1) Zhdankin, V. V. Hypervalent Iodine Chemistry Preparation, Structure and Synthetic Applications of Polyvalent Iodine Compounds, Wiley, Chichester 2013.

(2) (a) Hypervalent Iodine Chemistry. Modern Developments in Organic Synthesis, Wirth, T. (Ed.), Top. Curr. Chem. 224, Springer, Berlin, 2003. (b) Stang, P. J.; Zhdankin, V. V. Chem. Rev. 1996, 96, 1123. (c) Zhdankin, V. V.; Stang, P. J. Chem. Rev. 2002, 102, 2523. (d) Zhdankin, V. V.; Stang, P. J. Chem. Rev. 2008, 108, 5299. (e) Yoshimura, A.;

Zhdankin, V. V. Chem. Rev. 2016, 116, 3328. (f) Singh, F. V.; Wirth, T.; Chem. Asian J. 2014, 9, 950 .

(3) (a) Richardson, R. D.; Wirth, T. Angew. Chem. Int. Ed. 2006, 45, 4402. (b) Ochiai, M.; Miyamoto, K. Eur. J. Org. Chem. 2008, 4229. (c) Ochiai, M. Chem. Rec. 2007, 7, 12. (d) Dohi, T.; Kita, Y. Chem. Commun. 2009, 2073. (e) Uyanik, M.; Ishihara, K. Chem. Commun. 2009, 2086. (f) Yusubov, M. S.; Zhdankin, V. V. Reef. Tech. 2015, 1, 49.

(4) Souto, J. A.; Martínez, C.; Velilla, I.; Muñiz, K. Angew. Chem. Int. Ed. 2013, 52, 1324.

(5) For work on related compounds bearing nitrogen groups such as phthalimide and saccharin: (a) Yoshimura, A.; Koski, S. R.; Fuchs, J. M.; Saito, A.; Nemykin, V. N.; Zhdankin, V. V. Chem. Eur. J. 2015, 21, 5328. (b) Kiyokawa, K.; Kosaka, T.; Kojima, T.; Minakata, S. Angew. Chem. Int. Ed. 2015, 54, 13719. (c) Hadjiaraooglou, L.; Spyroudis, S.; Varvoglis, A. Synthesis 1983, 207. (d) Papadopoulou, M.; Varvoglis, A. Chem. Res. Synop. 1983, 66. (e) Papadopoulou, M.; Varvoglis, A. Chem. Res. Synop. 1984, 166.

(6) For related bromine(III) reagents incorporating transferable nitrogen groups: (a) Ochiai, M.; Kaneaki, T.; Tada, N.; Miyamoto, K.; Chuman, H.; Shiro, M.; Hayashi, S.; Nakanishi, 
W. J. Am. Chem. Soc. 2007, 129, 12938. (b) Ochiai, M.; Miyamoto, K.; Kaneaki, T.; Hayashi, S.; Nakanishi, W. Science 2011, 332, 448.

(7) For the alternative concept of electrophilic amination with hypervalent iodine reagents:

(a) Tellitu, I.; Domínguez, E. Trends Heterocycl. Chem. 2011, 15, 23. (b) Narayan, R.;

Manna, S.; Antonchick, A. P. Synlett 2015, 26, 1785. (c) Samanta, R.; Matcha, K.; Antonchick, A. P. Eur. J. Org. Chem. 2013, 5769.

(8) Souto, J. A.; Zian, D.; Muñiz, K. J. Am. Chem. Soc. 2012, 134, 7242.

(9) Kiyokawa, K.; Yahata, S.; Kojima, T.; Minakata, S. Org. Lett. 2014, 16, 4646.

(10) Souto, J. A.; Becker, P.; Iglesias, A.; Muñiz, K. J. Am. Chem. Soc. 2012, 134, 15505.

(11) Fra, L.; Millán, A.; Souto, J. A.; Muñiz, K. Angew. Chem. Int. Ed. 2014, 53, 7349.

(12) Moriyama, K.; Ishid, K.; Togo, H. Chem. Commun. 2015, 51, 2273.

(13) Souto, J. A.; González, Y.; Iglesias, Á.; Zian, D.; Lishchynskyi, A.; Muñiz, K. Chem. Asian J. 2012, 7, 1103.

(14) (a) Röben, C.; Souto, J. A.; González, Y.; Lishchynskyi, A.; Muñiz, K. Angew. Chem. Int. Ed. 2011, 50, 9478. (b) Röben, C.; Souto, J. A.; Escudero-Adán, E. C. Muñiz, K. Org. Lett. 2013, 15, 1008.

(15) (a) Martínez, C.; Muñiz, K. J. Org. Chem. 2013, 78, 2168. (b) Cardona, F.; Goti, A. Nature Chem. 2009, 1, 269. (c) de Figueiredo, R. M. Angew. Chem. Int. Ed. 2009, 48, 1190. (d) De Jong, S.; Nosal, D. G.; Wardrop, D. J. Tetrahedron 2012, 68, 4067.

(16) For an overview on alkene difunctionalization with hypervalent iodine(III) reagents: Romero, R. M.; Wöste, T. H.; Muñiz, K. Chem. Asian J. 2014, 9, 972.

(17) Funes-Ardoiz, I.; Sameera, W. M. C.; Romero, R. M.; Martínez, C.; Souto, J. A.; Sampero, D.; Muñiz, K.; Maseras, F. Chem. Eur. J. 2016, 22, 7545.

(18) See Supporting Information for details. 
(19) Haubenreisser, S.; Wöste, T. H.; Martínez, C.; Ishihara, K.; Muñiz, K. Angew. Chem. Int. Ed. 2016, 55, 413.

(20) Ochiai, M. in Chemistry of Hypervalent Compounds (Ed.: K. Akiba), Wiley-VCH, New York, 359, 1999.

(21) Uyanik, M.; Ishihara, K. J. Synth. Org. Chem. Jpn. 2012, 70, 1116.

(22) (a) Kong, W.; Feige, P.; de Haro, T.; Nevado, C. Angew. Chem. Int. Ed. 2013, 52, 2469. (b) Fujita, M.; Yoshida, Y.; Miyata, K.; Wakisaka, A.; Sugimura, T. Angew. Chem. Int. Ed. 2010, 49, 7068. (c) Fujita, M.; Wakita, M.; Sugimura, T. Chem. Commun. 2011, 47, 3983. (d) Uyanik, M.; Yasui, T.; Ishihara, K. Angew. Chem. Int. Ed. 2010, 49, 2175. (e) Farid, U.; Wirth, T. Angew. Chem. Int. Ed. 2012, 51, 3462. (f) Mizar, P.; Wirth, T. Angew. Chem. Int. Ed. 2014, 53, 5993. 